\section{Long-term outcome of combined trabeculotomy- trabeculectomy by a single surgeon in patients with primary congenital glaucoma}

D Sood ${ }^{1,2}$, A Rathore ${ }^{1}$, I Sood ${ }^{2}$, G Singh ${ }^{1}$ and NN Sood ${ }^{1,2}$
${ }^{1}$ Glaucoma Clinic New Delhi, New Delhi India

\section{${ }^{2}$ SK Glaucoma Care Foundation, New Delhi India}

Correspondence: D Sood, Glaucoma Clinic New Delhi, P-13, South Extension Part 2, New Delhi 110049 India

Tel: +(011) 2625

7803/26252000;

Fax: +(011) 26265409.

E-mail: drsood@

glaucomadelhi.in

Received: 7 February 2017 Accepted in revised form: 3 August 2017 Published online: 6 October 2017

\begin{abstract}
Purpose Assess long-term outcome of combined trabeculotomy-trabeculectomy (CTT) in primary congenital glaucoma (PCG). Methods Data of PCG patients treated with CTT by a single surgeon between 1976 and 1993 were reviewed for reduction in intraocular pressure (IOP), visual acuities, surgical success rates, and need for repeat surgeries at last follow-up (FU). At the last FU, IOP $<21 \mathrm{~mm} \mathrm{Hg}$ without any topical antiglaucoma medication (TAM) was complete success and with 1-2 TAMs was qualified success. IOP reduction $\geq 30 \%$ without any TAM was modified complete success and with 1-2 TAMs was modified qualified success. IOP $>21 \mathrm{~mm} \mathrm{Hg}$, IOP reduction $<30 \%$, or use of $>2$ TAM at last $\mathrm{FU}$, or need for additional surgery was considered as failure.

Results Two hundred thirty eyes of $\mathbf{1 2 1}$ patients had been followed up for 21.5-38

\section{Introduction}

The most prevalent glaucoma in the paediatric population is primary congenital glaucoma (PCG), also called isolated goniodysgenesis. ${ }^{1,2}$ In PCG, the intraocular pressure (IOP) increases due to isolated maldevelopment of the ocular trabecular meshwork or the angle of the anterior chamber with no other developmental anomaly in the eye or any systemic congenital anomaly. ${ }^{1-3}$ Most cases are sporadic. Due to autosomal recessive transmission, the incidence may be much higher in groups where consanguineous marriages are common. Generally, PCG becomes apparent at birth or early childhood ( $<3$ years of age) ${ }^{2}$

This study assesses the long-term outcome of combined trabeculotomy-trabeculectomy (CTT) performed by a single surgeon in PCG patients seen over a mean period of 28.9 years.
\end{abstract} years (mean $28.87 \pm 2.77$ years). Eyes that were pthisical (3), had immeasurable IOP (2), or IOP $<6 \mathrm{~mm} \mathrm{Hg}$ (3) were excluded from the success analysis. At last FU, mean IOP reduction was $22.71 \pm 11.28 \mathrm{~mm} \mathrm{Hg}$ and TAM score was $1.71(0-4)$. Complete success was achieved in $14(6.3 \%)$, modified complete success in $14(6.3 \%)$, qualified success in 148 $(66.7 \%)$, and modified qualified success in 140 (63.1\%). Success probability was $95 \%$ till 25 years and $92,90,85,79$ and $68 \%$ at $26,27,28$, 29 , and 30 years, respectively. Severe visual impairment or functional blindness was found in only $13(10.7 \%)$ patients.

Conclusion CTT shows long-term success in PCG patients. All patients must be monitored for IOP control to avoid need for repeat surgeries. Eye (2018) 32, 426-432; doi:10.1038/eye.2017.207; published online 6 October 2017

\section{Materials and methods}

We analysed the medical records of all patients with PCG who had been treated with CTT performed by a single surgeon between 1976 and 1993 at a tertiary glaucoma centre in India. The study adhered to the tenets of the Declaration of Helsinki with appropriate safeguards for patient confidentiality, after approval from the Ethics Committee.

\section{Examination procedure}

Intraocular pressure Preoperative IOP had been measured with a Perkin's handheld applanation tonometer during the early phase of anaesthesia. In the postoperative follow-up (FU) period, IOP was measured by Goldmann applanation 
tonometer in patients who cooperated for a slit-lamp examination.

Visual function Quantitative assessment of visual function was not possible in the preoperative phase as the children were too young. During the postoperative and FU periods, Snellens chart was used to assess visual function.

\section{Review of data}

Gender, right or left eye, consanguinity, family history of glaucoma, and the presence of systemic illnesses were noted. We noted the category of PCG diagnosed. We also noted the corneal diameter and clarity at presentation, the preoperative IOP and the age at surgery.

For each patient, we noted the total number of years of FU and whether any repeat surgery was needed after the first glaucoma surgery done at our centre. Other parameters reviewed included the visual acuity, presence of bleb, IOP, the number of topical antiglaucoma medications (TAMs) being used, the visibility of the optic nerve head, and the refractive error at the last FU.

Patients were excluded from the study if they had a secondary paediatric glaucoma or an acquired diagnosis, $<20$ years of FU, incomplete FU, or initial surgery by another surgeon. We also excluded patients whose preoperative data were not available.

\section{Outcome measures}

We based our diagnosis of PCG on increased corneal diameter, loss of corneal transparency/Haab's striae, photophobia, and raised IOP. Outcome measures analysed included reduction in IOP, visual acuities, surgical success rates, and the need for repeat surgeries.

\section{Surgical technique}

All the surgeries were done by a single surgeon (NNS). Combined trabeculotomy-trabeculectomy (CTT) was done in all patients as the primary procedure. Where needed, bilateral surgery was performed during the same sitting to avoid repeated anaesthesia. Under general anaesthesia and aseptic technique, a limbus-based conjunctival flap was raised. A partial thickness

triangular scleral flap was dissected. The Schlemm's canal was then dissected and trabeculotomy ab externo (Harms trabeculotome) was performed on either side of the radial incision, 180 degrees of the angle was treated. Then, trabeculectomy was performed and the scleral flap was sutured back. After completing the surgery on the first eye, the surgeon operated on the second eye using similar technique but with a new set of instruments, drapes, drops, gown, gloves, and so on.

\section{Success criteria}

Success rates were defined prior to data analysis. Complete success was defined as an IOP $<21 \mathrm{~mm} \mathrm{Hg}$ without any TAM at the last FU. Qualified success was defined as an IOP $<21 \mathrm{~mm} \mathrm{Hg}$ with $1-2$ additional TAM at the last FU. Failure was defined as an IOP $>21 \mathrm{~mm} \mathrm{Hg}$ with or use of more than two TAM at the last FU, or the need for additional surgery. A modified classification was also used. Modified complete success was considered if $30 \%$ drop in IOP was achieved without any TAM.

Modified qualified success was considered if target IOP was achieved with 1-2 TAM. Failure was considered if more than two TAMs were used or the target IOP was not achieved. The need for a repeat glaucoma surgery or diode laser transscleral cyclophotocoagulation was also considered as failure.

\section{Statistical analysis}

All statistical analysis was performed by MS-Excel 2007 and SPSS-16 (SPSS Inc. Released 2007. SPSS for Windows, Version 16.0. Chicago, SPSS Inc., Chicago, IL, USA) software. Quantitative variables were expressed as mean and standard deviation (SD) and qualitative variables by frequencies and percentages. Chi-square test examined the association between two or more qualitative variables. Student $t$-test was used for comparison of quantitative data. Logistic regression analysis was used for predicting the better prognostic factor. For all statistical analysis, $P<0.05$ was considered as statistically significant. Kaplan-Meier survival analysis was done to estimate the cumulative success probability of the surgery.

\section{Results}

\section{Preoperative findings}

Complete data was available for 230 eyes of 121 patients. The patient characteristics are summarised in Table 1. There were 172 (75\%) eyes diagnosed with newborn glaucoma and 58 (25\%) eyes with infantile glaucoma. The affliction was bilateral in most cases (90\%).

None of the infants had any associated systemic illnesses. Family history of glaucoma was present in only $5 \%(6 / 121)$ of the patients. Consanguineous marriages in the family were also uncommon (seen in the case of only three of the patients). The mean preoperative IOP was $37.29 \pm 10.72 \mathrm{~mm} \mathrm{Hg}$ (range, 19-65 $\mathrm{mm} \mathrm{Hg}$ ) and the mean age of the patients at the primary CTT surgery was 
Table 1 Baseline demographic characteristics of 230 eyes of 121 patients with primary congenital glaucoma

\begin{tabular}{lcc}
\hline Characteristic & Parameter & Value \\
\hline Age at surgery $(n(\%))$ & Up to $28 \mathrm{~d}$ & $122(53.0)$ \\
& $28 \mathrm{~d}-3$ years & $108(47.0)$ \\
& $>3 \mathrm{y}$ & $0(0)$ \\
Gender $(N(\%))$ & Male & $81(66.9)$ \\
& Female & $40(33.1)$ \\
Affliction $(N(\%))$ & Unilateral & $12(9.9)$ \\
& Bilateral & $109(90.1)$ \\
Family history of glaucoma & $N(\%)$ & $6(5.0)$ \\
Consanguineous marriages in the family & $N(\%)$ & $3(2.5)$ \\
Associated systemic illness & $N(\%)$ & $0(0)$ \\
& Mean \pm SD (Range) & $13.10 \pm 1.38(10-17)$ \\
Corneal diameter at the time of surgery & $10-12 \mathrm{~mm}(n(\%))$ & $81(35.2)$ \\
& $13-14 \mathrm{~mm}(n(\%))$ & $112(48.7)$ \\
& $15-16 \mathrm{~mm}(n(\%))$ & $35(15.2)$ \\
Corneal transparency & $>16 \mathrm{~mm}(n(\%))$ & $2(0.9)$ \\
& Clear $(n(\%))$ & $66(28.7)$ \\
& Oedema $(n(\%))$ & $32(13.91)$ \\
& Haab's striae $(n(\%))$ & $90(39.13)$ \\
& Opacity $(n(\%))$ & $42(18.26)$ \\
\hline
\end{tabular}

Abbreviations: $\mathrm{d}$, days; $n$ (\%), number of eyes (percentage); $N(\%)$, number of patients (percentage); $\mathrm{y}$, years.

Table 2 Time to repeat surgery following primary CTT in 230 eyes

\begin{tabular}{|c|c|c|c|c|c|c|}
\hline $\begin{array}{l}\text { Time since primary } \\
\text { CTT }\end{array}$ & $\begin{array}{c}\text { 1st repeat surgery } \\
(n)\end{array}$ & $\begin{array}{l}\text { 2nd repeat surgery } \\
\text { (n) }\end{array}$ & $\begin{array}{l}3 r d \text { repeat surgery } \\
\text { (n) }\end{array}$ & $\begin{array}{c}\text { 4th repeat surgery } \\
\text { (n) }\end{array}$ & $\begin{array}{l}\text { 5th repeat surgery } \\
(n)\end{array}$ & $\begin{array}{c}\text { 6th repeat surgery } \\
(n)\end{array}$ \\
\hline Less than $1 y$ & 5 & 1 & 0 & 0 & 0 & 0 \\
\hline $1-5 y$ & 10 & 5 & 0 & 0 & 0 & 0 \\
\hline $5-10 y$ & 9 & 4 & 1 & 0 & 0 & 0 \\
\hline $10-20 y$ & 12 & 5 & 4 & 1 & 0 & 0 \\
\hline $20-30 y$ & 5 & 1 & 1 & 1 & 2 & 1 \\
\hline $30-40 y$ & 0 & 0 & 0 & 0 & 0 & 0 \\
\hline
\end{tabular}

Abbreviations: CTT, combined trabeculotomy-trabeculectomy; $n$, number of eyes; $\mathrm{y}$, year.

$51.3 \pm 61.6$ (4-300) days. The 165 eyes with PCG-related corneal haze had a mean IOP of $38.1 \mathrm{~mm} \mathrm{Hg}$ (19-65 mm Hg), while the 65 eyes with clear cornea had a mean IOP of $35.3 \mathrm{~mm} \mathrm{Hg}$ (21-61 $\mathrm{mm} \mathrm{Hg}$ ). Preoperative visualisation of the angle outflow structures on gonioscopy was not possible in most cases.

\section{Surgery algorithm}

CTT was the primary surgery done on all 230 eyes. In 53\% of cases, it was done within 28 days of birth. The first repeat surgery was done in $41(17.8 \%)$ eyes at a mean of $111.32 \pm 95.78$ months after the first surgery. Second and third repeat surgeries were required in 16 (mean of 103.3 months after primary CTT) and six eyes (mean of 173.3 months after primary CTT), respectively. Most common repeat surgery was trabeculectomy with mitomycin C (TMMC). Two eyes needed a fourth surgery after a mean duration of 241.5 months from primary surgery; one was a TMMC while the other was a cyclocryotherapy. A fifth intervention was needed in only two eyes (mean of 276 months after primary CTT). Cyclocryotherapy was repeated in one while the other needed an Ahmed glaucoma valve (AGV). At 335 months, a sixth surgery was also needed in the eye undergoing cyclocryotherapy and an AGV was implanted.

\section{Time of repeat surgery}

In the first year since the primary CTT, five eyes needed a second surgery while one eye needed a third surgery (Table 2). Only 11/68 repeat surgeries were needed after 20 years following the primary surgery.

\section{Findings at the last follow-up visit}

The FU period ranged from 21.5 years to 38 years (mean $28.87 \pm 2.77$ years). Three of the eyes had undergone atrophy, the IOP was immeasurable in three and $<6 \mathrm{~mm} \mathrm{Hg}$ in two. These eight eyes were excluded from the analysis of success based on IOP or medication scores. 
For the 222 eyes analysed, the mean IOP at the last FU was $14.64 \pm 3.17 \mathrm{~mm} \mathrm{Hg}(6-32 \mathrm{~mm} \mathrm{Hg})$, mean reduction in IOP was $22.71 \pm 11.28 \mathrm{~mm} \mathrm{Hg}$ ( 1 to $55 \mathrm{~mm} \mathrm{Hg}$ ) and average percentage reduction in IOP was $57.44 \%$ $(4.76-86.67 \%)$. IOP was reduced by at least $30 \%$ in $208 / 222(93.7 \%)$ eyes. The TAM score in these 222 eyes was $1.71(0-4)$. Twenty eyes $(9 \%)$ did not require any TAM, 166 eyes $(74.8 \%)$ required $1-2$ TAMs, and 36 $(16.2 \%)$ required more than two TAMs.

At the last FU visit, the visual acuity assessed by the Snellen chart was available for all 230 eyes. Out of the 230 eyes, $66(28.7 \%)$ had best corrected visual acuity $(B C V A) \geq 20 / 40$ in the affected eye and 109 (47.4\%) had BCVA of 20/40-20/200. Fifty-five eyes (23.9\%) had BCVA $<20 / 200$.

There was a statistically significant association between the preoperative corneal diameter $(P=0.014)$ and the visual acuity at the last FU (Table 3 ). The association between preoperative corneal clarity and visual acuity at the last FU was also significant $(P=0.013)$.

\section{Success rates}

Success analysis was done for 222 of the 230 eyes. On the basis of predefined success criteria, complete success was achieved in 14 eyes (6.3\%) and qualified success in 148 $(66.7 \%)$ eyes (Table 4$)$. Additional glaucoma surgery was needed in 33 eyes considered for success analysis.

Modified complete success was achieved in 14 eyes, modified qualified success in 140 (63.1\%) eyes, and failure occurred in 68 (30.6\%) eyes. Kaplan-Meier survival analysis (Figure 1) revealed success probability of 95\% till 25 years and then it was $92,90,85,79$ and $68 \%$ at 26,27 , 28,29 , and 30 , years respectively.

\section{Bleb characteristics}

At the last FU, diffuse, low to moderately cystic blebs were present in $8(3.5 \%)$ eyes, while the blebs were flat in $222(96.5 \%)$ eyes.

\section{Optic nerve head}

The optic nerve head could not be viewed in $112 / 230$ eyes $(48.7 \%)$ at last FU because of poor corneal clarity. The vertical cup to disc ratio was $\leq 0.6$ in $77 / 230$ eyes $(33.5 \%)$ and $>0.6$ in $41 / 230$ eyes (17.8\%).

\section{Refractive errors}

Data for refractive errors at the last FU was available for all 230 eyes. Eighty (34.8\%) eyes were myopic and nine $(3.9 \%)$ eyes were hyperopic.
Table 3 Correlation between preoperative factors and the visual acuity in eyes with primary congenital glaucoma $(n=230)$

\begin{tabular}{|c|c|c|c|c|c|}
\hline \multirow[t]{2}{*}{ Parameters } & \multicolumn{3}{|c|}{ Visual acuity } & \multirow{2}{*}{$\begin{array}{c}\text { Total } \\
\text { number of } \\
\text { eyes }\end{array}$} & \multirow[t]{2}{*}{ P-value } \\
\hline & \multicolumn{3}{|c|}{$20 / 40$} & & \\
\hline \multicolumn{6}{|c|}{ Pre-operative IOP $(\mathrm{mm} \mathrm{Hg})$} \\
\hline$<20$ & 0 & 0 & 2 & 2 & \multirow[t]{6}{*}{0.301} \\
\hline $20-30$ & 9 & 20 & 11 & 40 & \\
\hline $30-40$ & 30 & 46 & 26 & 102 & \\
\hline $40-50$ & 21 & 24 & 8 & 53 & \\
\hline$>50$ & 8 & 15 & 10 & 33 & \\
\hline Total & 68 & 105 & 57 & 230 & \\
\hline \multicolumn{6}{|c|}{ Corneal diameter (at time of surgery) } \\
\hline $10-12 \mathrm{~mm}$ & 30 & 43 & 8 & 81 & \multirow[t]{5}{*}{$0.014^{*}$} \\
\hline $13-14 \mathrm{~mm}$ & 29 & 48 & 35 & 112 & \\
\hline $15-16 \mathrm{~mm}$ & 9 & 14 & 12 & 35 & \\
\hline$>16$ & 0 & 0 & 2 & 2 & \\
\hline Total & 68 & 105 & 57 & 230 & \\
\hline \multicolumn{6}{|l|}{ Diagnosis (n) } \\
\hline $\begin{array}{l}\text { New born } \\
\text { glaucoma }\end{array}$ & 49 & 83 & 40 & 172 & \multirow[t]{3}{*}{0.543} \\
\hline $\begin{array}{l}\text { Infantile } \\
\text { glaucoma }\end{array}$ & 19 & 22 & 17 & 58 & \\
\hline Total & 68 & 105 & 57 & 230 & \\
\hline \multicolumn{6}{|c|}{ Corneal clarity (n) } \\
\hline Clear & 28 & 28 & 10 & 66 & \multirow{5}{*}{$0.013^{*}$} \\
\hline $\begin{array}{l}\text { Haab's } \\
\text { striae }\end{array}$ & 26 & 39 & 25 & 90 & \\
\hline Oedema & 10 & 17 & 5 & 32 & \\
\hline Opacity & 4 & 21 & 17 & 42 & \\
\hline Total & 68 & 105 & 57 & 230 & \\
\hline
\end{tabular}

${ }^{*} P$ value was significant $(P<0.05)$.

Table 4 Success rate of combined trabeculotomy-trabeculectomy in 222 eyes with primary congenital glaucoma

\begin{tabular}{lcc}
\hline Success criteria & No. of eyes & Percentage \\
\hline Based on IOP at last follow-up & & \\
Complete success & 14 & 6.3 \\
Qualified success & 148 & 66.7 \\
Failure & 60 & 27 \\
& & \\
Based on percentage reduction in IOP at last follow up & \\
Modified complete success & 14 & 6.3 \\
Modified qualified success & 140 & 63.1 \\
Modified failure & 68 & 30.6 \\
\hline
\end{tabular}

\section{Discussion}

Treatment of PCG focuses on removing the obstruction to aqueous flow due to structural abnormalities in the anterior chamber. An internal approach such as goniotomy or an external approach such as trabeculotomy or trabeculectomy may be tried. Unlike 
a

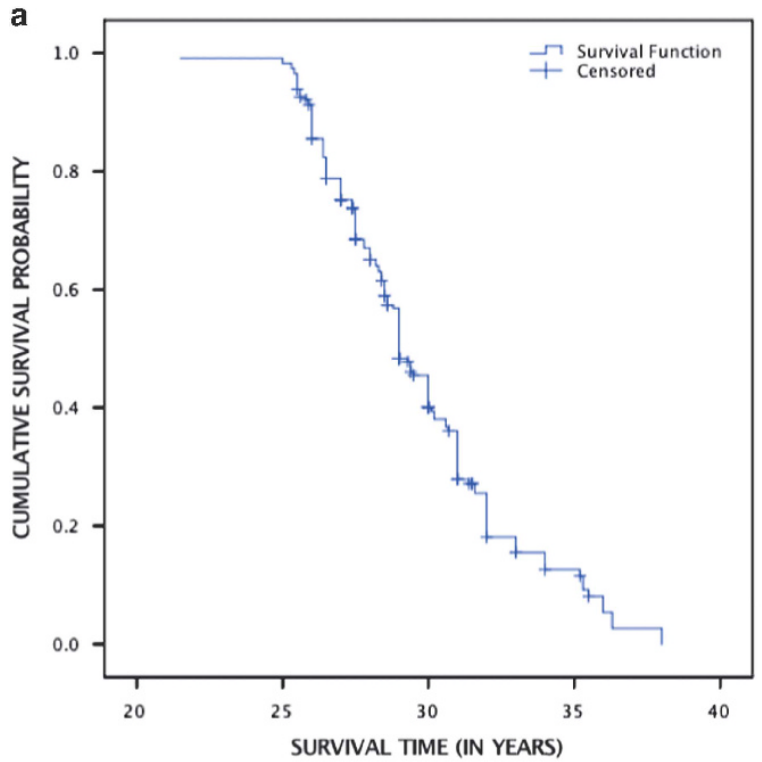

b

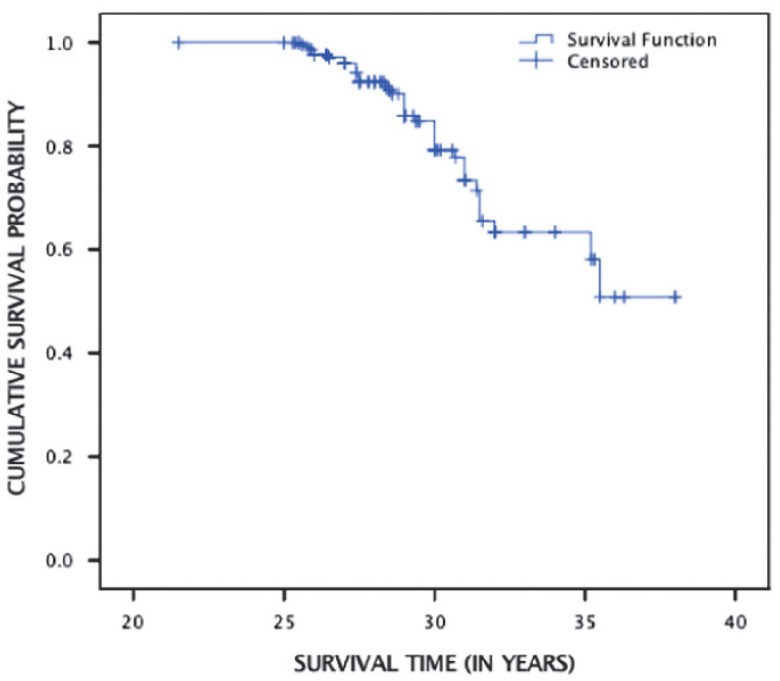

Figure 1 Kaplan-Meier survival analysis charts showing cumulative success probability of eyes with complete success (a) and complete plus qualified success (b).

goniotomy, trabeculotomy and trabeculectomy can be done even when the cornea is cloudy and the glaucoma is advanced. 4

In 1983, NNS had found that congenital glaucoma in Indian children was commonly associated with corneal opacity and evaluated the efficacy of various surgical techniques.5,6 During the early 1970 s, NNS had tried goniotomy, goniopuncture, Scheie's operation, anterior sclerectomy, and iridencleisis to treat congenital glaucoma. Though there was reduction in IOP in most cases in the immediate postoperative period, the IOP started increasing with time and the success was no more than $55.5 \%$. He tried trabeculectomy in 55 eyes and got slightly better results of $63.4 \%$. Later, he tried CTT (which was a newer technique at that time) on 19 eyes. Seventeen eyes $(89.5 \%)$ had well controlled IOP at a FU ranging between 6 months and 2 years. ${ }^{6}$ He concluded that CTT was safe and effective in controlling IOP in the congenital glaucoma cases he operated upon and made it the standard for patients with newborn and infantile types of PCG at his glaucoma center.

Mandal et $\mathrm{al}^{7}$ had reported the surgical outcome of primary CTT by a single surgeon in 624 eyes of 360 consecutive patients with PCG. However, the mean FU period in our study is longer and the success rate higher.

Clinical criteria to establish a diagnosis of PCG include an IOP of $>21 \mathrm{~mm} \mathrm{Hg}$ in one or both eyes, infantile buphthalmos, increased corneal diameter, isolated angle anomalies and mild congenital iris anomalies. ${ }^{8}$ However, the angle structures could not be adequately evaluated because of corneal haziness and opacities. ${ }^{5}$ Subcategories are based on the age at onset. In our case series of 230 cases, there were 172 newborn glaucoma (0-1 month), 58 infantile glaucoma ( $>1-24$ months) and no late onset PCG ( $>2$ years). The type of PCG did not significantly affect the visual acuity at last FU.

PCG is typically bilateral with $25-30 \%$ being unilateral. ${ }^{1,2,4,9-12}$ However, as in another report, ${ }^{13}<10 \%$ of our cases were unilateral. Some studies report an even sex distribution ${ }^{4,9,11}$ but most report a male preponderance ${ }^{1,2,10,12,14}$ as seen in our study. Higher rate of consanguinity is associated with a more severe disease and poorer outcomes with surgery. ${ }^{13}$ At just $2.5 \%$, the rate of consanguinity in our patients was rather low.

During CTT, the trabeculotomy procedure cut through the total thickness of the trabecular mass and helped create a direct continuity between the anterior chamber and Schlemm's canal. Trabeculectomy created a supplementary fistula and the aqueous humour could bypass Schlemm's canal to be drained out of the anterior chamber. Further, CTT is helpful in those cases where visualisation of the angle becomes difficult due to corneal haziness ( $71 \%$ of the eyes in this study).

CTT showed good long-term success rates with either complete or qualified success in 162/222 (73.0\%) eyes over a mean of 28.9 years of FU, good control of IOP (at least $30 \%$ reduction at last FU in $93.7 \%(208 / 222)$ of the eyes) and a low rate of new surgical interventions (only $41 / 230$ eyes, $17.8 \%$ required repeat surgeries). In other studies, surgical success rates varied between 65 and $90 \% .{ }^{11,12,15-18}$ Such a wide variance in the success rates could be due to the difference in the number of eyes studied, the length of FU, and the definitions for surgical success. In addition, in some of these studies, CTT was augmented by an adjunctive antimetabolite such as 5-fluorouracil. As a result, success rates in some studies may have been higher than ours. 
Our study found a success probability of $95 \%$ till 25 years and then of $92,90,85,79$, and $68 \%$ at $26,27,28,29$, and 30 years, respectively, which is better than several other studies. ${ }^{12,18,19}$

There was no major difference in the mean preoperative IOP of the eyes with or without corneal haze. The postoperative visual acuity, however, seemed to be significantly affected by the degree of corneal clarity $(P=0.013)$. A recent study, however, reported more severe presentation but similar post management outcomes in eyes with PCG-related haziness as compared to eyes without haze. ${ }^{14}$

Preoperative visual acuity could not be checked in most of the children. Of the 230 eyes studied, $28.7 \%$ had a $\mathrm{BCVA} \geq 20 / 40$ at last FU, $47.4 \%$ had low vision (BCVA of $20 / 40-20 / 200$ ) and $23.9 \%$ with BCVA $<20 / 200$ were considered blind. However, World Health Organization currently defines visual impairment with respect to the BCVA of the better eye of the patient. ${ }^{20}$ At the last FU visit, out of the 121 patients, only $13(10.7 \%)$ had severe visual impairment or were functionally blind with BCVA $<20 / 200$ in the better eye. Over $47 \%$ had mild or no visual impairment and $42 \%$ had low vision with moderate visual impairment. Functional visual outcome was thus, much better than the encouraging results seen in earlier studies in Indian children.,12,19

In a study by Mandal et al, ${ }^{19}$ myopia was reported in over half the patients while a quarter had hyperopia. Dubey et $a l^{12}$ reported myopia in $76 \%$ of the eyes and hyperopia in $24 \%$. Our study reported only $34.8 \%$ of the eyes being myopic (24,>6 dioptres, 33 between 2 and 6 dioptres and 23 upto 2 dioptres) and $3.9 \%$ of the eyes being hyperopic (1,>6 dioptres, 5 between 2 and 6 dioptres and 3 upto 2 dioptres) at last FU. For the rest, refraction though attempted was limited by corneal opacities/Haab's striae. For optimum visual rehabilitation, refractive changes and amblyopia developing during the course of the disease need to be adequately managed. Amblyopia may develop due to corneal haze leading to stimulus deprivation or due to anisometropia. Usual treatment is by patching.

The study is limited by its retrospective design and the limited documentation of the postoperative changes in corneal diameter and cloudiness.

CTT is a viable option for initial procedure in PCG patients. However, all patients must be monitored for a long term for IOP control. Failure of adequate IOP control may necessitate repeat filtration surgeries or the use of glaucoma drainage devices. Optical correction of refractive errors occurring in the natural course of the disease and aggressive amblyopia therapy are also highly recommended.

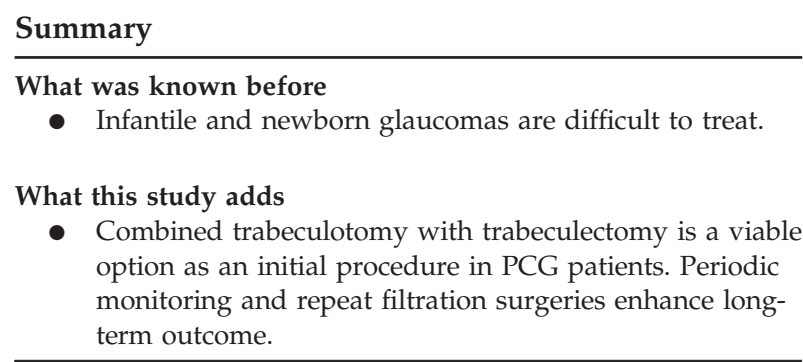

\section{Conflict of interest}

The authors declare no conflict of interest.

\section{Acknowledgements}

All combined trabeculotomy-trabeculectomies were done by Prof Narender Nath Sood while at the Dr. Rajendra Prasad Centre for Ophthalmic Sciences, AIIMS, New Delhi from where he retired in 1993. This study was funded by SK Glaucoma Care Foundation, New Delhi and PFC Pharma Focus India Pvt Ltd helped with the data analysis and preparation of the first draft of the manuscript.

\section{References}

1 Helmy H. Combined trabeculotomy-trabeculectomy versus Ahmed valve implantation for refractory primary congenital glaucoma in Egyptian patients: a long-term follow-up. Electron Physician 2016; 8: 1884-1891.

2 Mandal AK, Chakrabarti D. Update on congenital glaucoma. Indian J Ophthalmol 2011; 59: S148-S157.

3 Wu Z-K, Wu J, Tan Q, Jiang J, Song W-T, Xia X-B et al. Therapeutic effect analysis on the treatment of congenital glaucoma through modified combined trabeculotomytrabeculectomy. Int J Ophthalmol 2016; 9: 243-248.

4 Zagora SL, Funnell CL, Martin FJ, Smith JEH, Hing S, Bilson FA et al. Primary congenital glaucoma outcomes: lessons from 23 years of follow-up. Am J Ophthalmol 2015; 159(788-796): e2.

5 Agarwal HC, Sood NN, Kalra BR. Clinical presentation of congenital glaucoma. Indian J Ophthalmol 1983; 31: 619.

6 Sood NN, Aggarwal HC, Kalra BR. Evaluation of surgery for congenital glaucoma. Indian J Ophthalmol 1983; 31: 609.

7 Mandal AK, Gothwal VK, Nutheti R. Surgical outcome of primary developmental glaucoma: a single surgeon's longterm experience from a tertiary eye care centre in India. Eye 2007; 21: 764-774.

8 Glaucoma: Definitions and Classification. Am Acad Ophthalmol 2015. https:/ /www.aao.org/pediatric-centerdetail/glaucoma-definitions-classification. (accessed on 27 December 2016).

9 Sa Y, Er A-T. Surgical outcomes in children with primary congenital glaucoma: a 20-year experience. Eur J Ophthalmol 2016; 26: 581-587.

10 Kaur S. Glaucoma in childhood. Delhi J Ophthalmol 2014; 25: 160-166. 
11 Essuman VA, Braimah IZ, Ndanu TA, Ntim-Amponsah CT. Combined trabeculotomy and trabeculectomy: outcome for primary congenital glaucoma in a West African population Eye 2011; 25: 77-83.

12 Dubey S, Agrawal A, Chauhan L, Mukerjee S, Douglas G. Combined trabeculotomy-trabeculectomy with antimetabolite and releasable suture: outcome with primary congenital glaucoma in a north Indian population. Nepal J Ophthalmol 2015; 7: 16-25.

13 Kaur K, Mandal AK, Chakrabarti S. Primary congenital glaucoma and the involvement of CYP1B1. Middle East Afr J Ophthalmol 2011; 18: 7-16.

14 Li X, Mukkamala L, Origlieri CA, Holland BK, Fechtner RD, Khouri AS. Corneal haze as prognostic indicator of intraocular pressure in primary congenital glaucoma. J Glaucoma 2016; 25: e855-e860.

15 Al-Hazmi A, Awad A, Zwaan J, Al-Mesfer SA, Al-Jadaan I, Al-Mohammed A. Correlation between surgical success rate and severity of congenital glaucoma. Br J Ophthalmol 2005; 89: $449-453$.

16 Temkar S, Gupta S, Sihota R, Sharma R, Angmo D, Pujari A, Dada T. Illuminated microcatheter circumferential trabeculotomy versus combined trabeculotomytrabeculectomy for primary congenital glaucoma: a randomized controlled trial. Am J Ophthalmol 2015; 159(490-497): e2

17 Jalil A, Au L, Khan I, Ashworth J, Lloyd IC, Biswas S. Combined trabeculotomy-trabeculectomy augmented with 5-fluorouracil in paediatric glaucoma. Clin Experiment Ophthalmol 2011; 39: 207-214.

18 Campos-Mollo E, Moral-Cazalla R, Belmonte-Martínez J. [Combined trabeculotomy-trabeculectomy as the initial surgical procedure of primary developmental glaucoma]. Arch Soc Espanola Oftalmol 2008; 83: 479-485.

19 Mandal AK, Bhatia PG, Bhaskar A, Nutheti R. Long-term surgical and visual outcomes in Indian children with developmental glaucoma operated on within 6 months of birth. Ophthalmology 2004; 111: 283-290.

20 World Health Organization. Change the Definition of Blindness. http://www.who.int/blindness/Change $\% 20$ the $\% 20$ Definition \%20of\%20Blindness.pdf. (accessed on 2 January 2017). 